\title{
Impact of heavy-flavour production cross sections measured by the LHCb experiment on parton distribution functions at low $x$
}

\author{
PROSA Collaboration
}

\author{
O. Zenaiev ${ }^{1, a}$, A. Geiser ${ }^{1}$, K. Lipka ${ }^{1}$, J. Blümlein ${ }^{1}$, A. Cooper-Sarkar ${ }^{2}$, M.-V. Garzelli ${ }^{3}$, M. Guzzi ${ }^{4}$, O. Kuprash ${ }^{1}$,

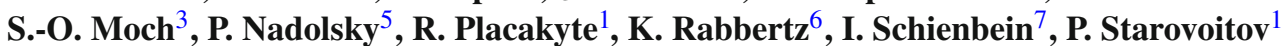 \\ ${ }^{1}$ DESY Hamburg and Zeuthen, Hamburg, Germany \\ ${ }^{2}$ University of Oxford, Oxford, UK \\ ${ }^{3}$ Universität Hamburg, Hamburg, Germany \\ ${ }^{4}$ School of Physics and Astronomy, The University of Manchester, Manchester, UK \\ ${ }^{5}$ Southern Methodist University, Dallas, TX, USA \\ ${ }^{6}$ Karlsruher Institut für Technologie, Karlsruhe, Germany \\ ${ }^{7}$ LPSC Grenoble, Grenoble, France
}

\begin{abstract}
The impact of recent measurements of heavyflavour production in deep inelastic $e p$ scattering and in $p p$ collisions on parton distribution functions is studied in a QCD analysis in the fixed-flavour number scheme at next-to-leading order. Differential cross sections of charmand beauty-hadron production measured by $\mathrm{LHCb}$ are used together with inclusive and heavy-flavour production cross sections in deep inelastic scattering at HERA. The heavyflavour data of the $\mathrm{LHCb}$ experiment impose additional constraints on the gluon and the sea-quark distributions at low partonic fractions $x$ of the proton momentum, down to $x \sim 5 \times 10^{-6}$. This kinematic range is currently not covered by other experimental data in perturbative QCD fits.
\end{abstract}

\section{Introduction}

Understanding the nucleon structure is one of the fundamental tasks of modern particle physics. In quantum chromodynamics (QCD), the structure of the nucleon is described by parton distribution functions (PDFs), which, in collinear factorisation, represent probability densities to find a parton of longitudinal fraction $x$ of the nucleon momentum at a factorisation scale $\mu_{f}$. The scale evolution of the PDFs is uniquely predicted by the renormalisation group equations for factorisation [1-9]. The $x$-dependence cannot be derived from first principles and must be constrained by experimental measurements. The precision of the PDFs is of key importance for interpreting the measurements in hadronic collisions. In

\footnotetext{
a e-mail: oleksandr.zenaiev@desy.de
}

particular, the uncertainty of the proton PDFs must be significantly reduced in order to improve the accuracy of theory predictions for Standard Model (SM) processes at the LHC.

Deep inelastic lepton-proton scattering (DIS) experiments cover a broad range in $x$ and $\mu_{f}$. In the perturbative regime, a wide $x$-range of $10^{-4}<x \lesssim 10^{-1}$ is probed by the data of the $\mathrm{H} 1$ and ZEUS experiments at the HERA collider [10]. These measurements impose the tightest constraints on the existing PDFs. However, additional measurements are necessary for a better flavour separation and to constrain the kinematic ranges of very small and very high $x$, where the gluon distribution is poorly known. A better constraint on the high- $x$ gluon is needed for an accurate description of the SM backgrounds in searches for new-particle production at high masses or momenta. A significant reduction of the uncertainty of the low- $x$ gluon distribution is important for studies of parton dynamics, non-linear and saturation effects. Furthermore, the precision of the gluon distribution at low $x$ has implications in physics of atmospheric showers, being crucial for cross-section predictions of high-energy neutrino DIS interactions [11] and for calculations of prompt lepton fluxes in the atmosphere [12].

Heavy-flavour measurements of the LHCb Collaboration $[13,14]$ at the LHC probe the very forward range of the heavy-hadron rapidity $y$ and are sensitive to the gluon PDF at low $x$, as schematically shown in Fig. 1. For this illustration, in the calculation of the kinematics of heavy-quark production at HERA, the leading-order (LO) relation is used for the typical gluon $x$ in boson-gluon fusion, $x=x_{B j}\left(1+\frac{4 m_{Q}^{2}}{Q^{2}}\right)$, 


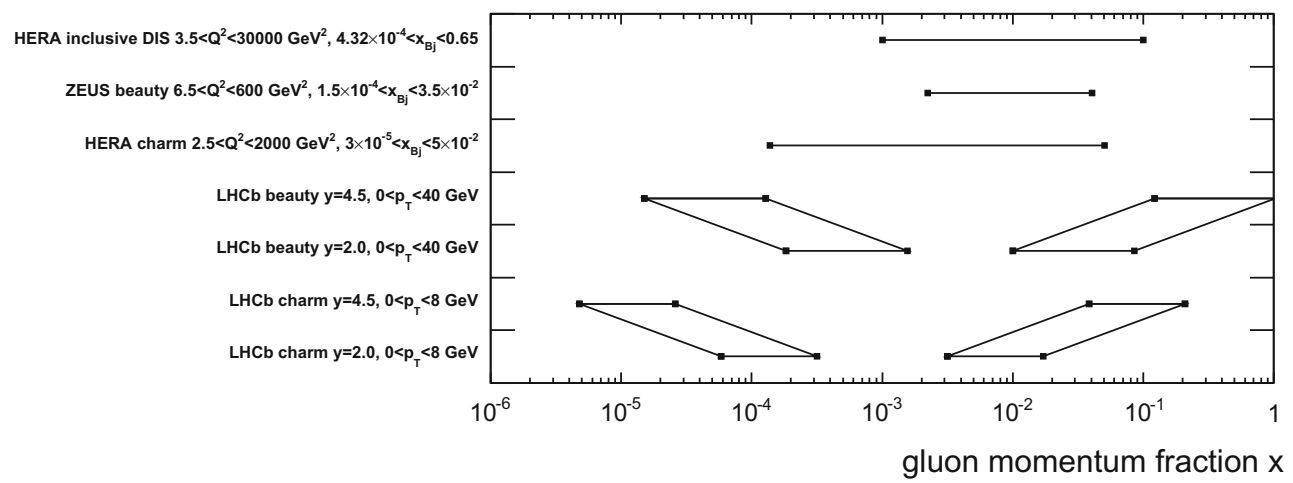

Fig. 1 Kinematic range in $x$ for the gluon density covered by measurements at HERA and LHCb. For the HERA inclusive DIS data, the $x$ range is indicated, where the gluon PDF uncertainties are less than
$10 \%$ at $\mu_{f}^{2}=10 \mathrm{GeV}^{2}$. For the LHCb data, the upper (lower) edge of the box refers to the indicated upper (lower) end of the rapidity, $y$, range of the heavy-hadron production where $x_{B j}$ denotes the Bjorken scaling variable, $m_{Q}$ is the heavy-quark mass, and $Q^{2}$ is the virtuality of the exchanged electroweak boson. In the case of heavy-quark production at LHCb, the LO formula $x=e^{ \pm y} \frac{\sqrt{p_{T}^{2}+m_{Q}^{2}}}{E_{p}}$, assuming $p_{z}=0$ in the parton-parton rest frame, is applied. Here, $p_{T}$ and $p_{z}$ represent the transverse and longitudinal momenta of the heavy quark, respectively, and $E_{p}$ is the proton beam energy.

Heavy-flavour production in proton-proton collisions at LHC is dominated by gluon-gluon fusion. Therefore the LHCb measurements of charm [13] and beauty [14] production in the forward region $2.0<y<4.5$ probe the gluon distribution at $5 \times 10^{-6} \lesssim x \lesssim 10^{-4}$, a region which is not accessible with HERA data. Note that the LHCb data are sensitive to the product of gluon densities in two nonoverlapping low and medium-to-high $x$ ranges, as illustrated in Fig. 1. Since the medium range is already well constrained by HERA data, which furthermore bridge the gap between the two LHCb ranges, the major impact of the LHCb heavyflavour measurements is expected at $5 \times 10^{-6} \lesssim x \lesssim 10^{-4}$. The advantage of using heavy-flavour data is that the charm and beauty masses provide hard scales for the perturbative QCD (pQCD) expansion all the way down to their production threshold. To estimate the impact of the LHCb measurement of charm and beauty production on the gluon distribution at low $x$, these data are included in a QCD analysis together with the inclusive DIS [10] and heavy-flavour production [15,16] cross sections measured at HERA.

\section{Experimental data used in the QCD analysis}

The main objective of the present QCD analysis is to demonstrate the constraining power of the measurements of heavyflavour production in DIS and $p p$ collisions for the determination of the PDFs of the proton. The measurements of charm and beauty production at HERA and $\mathrm{LHCb}$, together with the combined HERA inclusive cross-section measurements, are used in a next-to-leading order (NLO) pQCD analysis.

Neutral current (NC) and charged current (CC) inclusive DIS cross sections in ep scattering are directly sensitive to the valence- and sea-quark distributions and probe the gluon distribution through scaling violations [1-3]. HERA measurements of the NC and CC cross sections in DIS at a centre-of-mass energy $\sqrt{s}=320 \mathrm{GeV}$ have been combined taking into account systematic correlations [10]. This combined data set contains the complete information on inclusive DIS cross sections published by the H1 and ZEUS Collaborations based on data collected in the years 1994-2000, and has been used for the determination of the PDF set HERAPDF1.0 [10]. The kinematic range of the NC data is $6 \times 10^{-7} \leq x_{B j} \leq 0.65,0.045 \leq Q^{2} \leq 30000 \mathrm{GeV}^{2}$, however, in the original HERAPDF1.0 fit the $Q^{2}$ range of the data was restricted to $Q^{2}>Q_{\min }^{2}=3.5 \mathrm{GeV}^{2}$ to ensure the applicability of perturbative calculations [10]. The CC cross sections span the kinematic range of $1.3 \times 10^{-2} \leq x_{B j} \leq 0.40$ and $300 \leq Q^{2} \leq 30000 \mathrm{GeV}^{2}$. These combined NC $e^{ \pm} p$ and $\mathrm{CC} e^{ \pm} p$ cross sections represent the basis for all PDF determinations.

In ep scattering, charm and beauty quarks are produced predominantly in the photon-gluon fusion process which provides a direct probe of the gluon distribution in the proton. Measurements of open-charm production cross sections in DIS at HERA from the H1 and ZEUS Collaborations have been combined [15]. Cross sections for charm production were obtained in the kinematic range of $2.5 \leq Q^{2} \leq 2000$ $\mathrm{GeV}^{2}$ and $3 \times 10^{-5} \leq x_{B j} \leq 5 \times 10^{-2}$. The combination method accounts for the correlations of the systematic uncertainties among the different data sets. These combined measurements were used to improve constraints on the gluon distribution and to determine the charm-quark mass [15]. The charm reduced cross sections determined as a function of $Q^{2}$ and $x_{B j}$ are used in the present analysis together with all provided details on the systematic correlations. In addition, 
cross sections for the production of $b$ quarks in $e p$ scattering, as measured by the ZEUS Collaboration [16] are used in the present analysis. These data correspond to an integrated luminosity of $354 \mathrm{pb}^{-1}$ and cover the kinematic range of $5<Q^{2}<1000 \mathrm{GeV}^{2}$. The $b$ - and $c$-quark content in the events with at least one jet have been extracted using the invariant mass of charged tracks associated with secondary vertices and lifetime information, and the beauty-quark mass has been measured [16]. In the present analysis, the beautyquark production data are used mainly to improve constraints on the beauty-quark mass.

For additional constraints on the gluon distribution at low $x$ the differential cross sections of charm and beauty production in $p p$ collisions at $\sqrt{s}=7 \mathrm{TeV}$ from the $\mathrm{LHCb}$ experiment are used for the first time. The measurement of charm production [13] is based on data corresponding to an integrated luminosity of $15 \mathrm{nb}^{-1}$. Charm production is identified through the full reconstruction of decays of the charmed hadrons ${ }^{1} D^{0}, D^{+}, D^{*+}, D_{s}^{+}$and $\Lambda_{c}^{+}$. The cross sections are measured as a function of the transverse momentum, $p_{T}$, and rapidity, $y$, of the reconstructed hadrons. The $\mathrm{LHCb}$ data on $B$-meson production in $p p$ collisions [14] correspond to an integrated luminosity of $0.36 \mathrm{fb}^{-1}$. The $B^{+}, B^{0}$ and $B_{s}^{0}$ mesons are reconstructed in exclusive decays mainly involving $J / \psi$ final states. Correlations between the experimental systematic uncertainties are accounted for as described in the original publications. An uncorrelated systematic uncertainty is obtained for each distribution by subtracting the correlated uncertainties from the total ones. The $3.5 \%$ luminosity uncertainty is treated as correlated between the measurements of charm and beauty production. In the present analysis, the normalised cross sections, $\frac{\mathrm{d} \sigma}{\mathrm{d} y} / \frac{\mathrm{d} \sigma}{\mathrm{d} y_{0}}$, for charm and beauty production are calculated from the absolute measurements published by LHCb and are used in the QCD analysis, with $\frac{\mathrm{d} \sigma}{\mathrm{d} y_{0}}$ being the cross section in the center bin, $3<y<3.5$, of the measured rapidity range in each $p_{T}$ bin. The uncorrelated experimental uncertainty on $\frac{\mathrm{d} \sigma}{\mathrm{d} y_{0}}$ is propagated as a correlated uncertainty to the respective complementary rapidity bins. The QCD analysis is performed by using both, absolute or normalised, representations of the LHCb measurements, alternatively.

\section{Theoretical predictions for heavy-flavour production}

In the QCD analysis, the experimental measurements are confronted with corresponding theoretical predictions. Complete fixed-order theoretical predictions for differential production of charm and beauty in both $e p$ and $p p$ collisions only exist at NLO in the fixed-flavour number scheme (FFNS). For higher orders, the most comprehensive results for heavy-

$\overline{1}$ Charge conjugation is always implied for charm and beauty hadrons. quark production in $e p$ collisions are given in [17], which contain combined approximate next-to-next-to-leading order (NNLO) $O\left(\alpha_{s}^{3}\right)$ expressions for three kinematic limits: in the limit of high partonic centre-of-mass energy squared, in the region of the production threshold, and in the high-scale region. For heavy quark-pair production in $p p$ collisions, the cross sections in single-particle kinematics have been calculated at approximate NNLO $O\left(\alpha_{s}^{4}\right)[18,19]$ and at approximate $\mathrm{N}^{3} \mathrm{LO}$ [20] by using methods of threshold resummation beyond the leading logarithmic approximation. Further theory developments are necessary to include the available HERA and LHCb heavy-flavour production measurements in a QCD analysis at NNLO.

Consistent with the necessary theory predictions, the presented QCD analysis is performed at NLO in FFNS. This scheme and its applicability to HERA measurements is discussed in detail in Ref. [15] and references therein. Predictions for HERA data are obtained by following the approach of the ABM group at NLO using its implementation in OPENQCDRAD [21-23] in the framework of HERAFitter [24]. The number of active flavours is set to $N_{f}=3$, and the renormalisation and factorisation scales (pQCD scales) for heavy-flavour production are chosen as $\mu_{r}=\mu_{f}=$ $\sqrt{Q^{2}+4 m_{Q}^{2}}$, where $m_{Q}$ denotes the pole mass of $c$ or $b$ quarks. $^{2}$ For the light-flavour contribution to the inclusive DIS cross sections, the pQCD scales are set to $\mu_{r}=\mu_{f}=Q$.

Theoretical predictions for heavy-quark production in $p p$ collisions are obtained using the massive NLO calculations [25-27] in the FFNS, also available as part of the Mangano-Nason-Ridolfi (MNR) calculations [28]. The pQCD scales are chosen as $\mu_{r, f}=A_{r, f}^{c, b} \mu_{0}$, with $\mu_{0}=$ $\sqrt{p_{T}^{2}+m_{Q}^{2}}$ and $A_{r, f}^{c, b}$ being coefficients for $c$ and $b$ quarks, which are discussed in the following. These predictions were used successfully for beauty production in $p \bar{p}$ collisions at the $S p \bar{p} S$ [29-31] and the Tevatron ${ }^{3}$ [32]. They are conceptually very similar to the Frixione-Mangano-Nason-Ridolfi (FMNR) predictions [36,37] employed for heavy-flavour photoproduction at HERA $[38,39]$.

The cross-section predictions for heavy-flavoured hadron production not only depend on the kinematics of the heavy-

\footnotetext{
2 The pole mass is used for consistency with the $p p$ predictions, since $\overline{\mathrm{MS}}$ running mass predictions are not available for $\mathrm{LHCb}$.

3 Provided that fragmentation and other uncertainties are properly treated [33]. Note that the NLO+NLL (FONLL) [34] calculations used there, and also used by $\mathrm{LHCb}[13,14]$, slightly reduce the cross sections at high transverse momenta with respect to the pure NLO calculation, while they are identical at low transverse momenta [34]. The Tevatron data are well described by FONLL [33]. This conclusion is also applicable to the NLO calculations [27] used here, since these were used as input for the NLO part of the FONLL calculations. The claim in [32] that the NLO predictions undershoot the data while FONLL describes them must thus be attributed to parametrisations beyond the perturbative part of the calculations, as illustrated, e.g., in Fig. 7 of [35].
} 
flavour production mechanism, but also on the fragmentation of the heavy quark into a particular final-state hadron. There is no final-state factorisation scale in the FFNS since collinear logarithms of the heavy-quark mass are included in fixedorder perturbation theory. The calculations in [25-27,40] describe the production of an on-shell heavy quark. Near the kinematic threshold, the transition of the heavy quark into the observed heavy-flavoured hadron can be taken into account by multiplying the cross section with the appropriate fragmentation fraction. This leads to an excellent description of $B$ - and $D$-meson production measurements at the Tevatron and the LHC from $p_{T}=0$ up to $p_{T} \sim 4 m_{Q}[41,42]$. The scope of these calculations can be extended by convoluting the heavy-quark production cross section with a suitable scale-independent fragmentation function describing the hadronisation of the heavy quark. The implementation of the convolution is not unique once the quark and hadron masses are taken into account, and leads to a potentially $p_{T}$-dependent modeling uncertainty which is, however, small compared to the scale-choice uncertainty at NLO. This fragmentation function is used on a purely phenomenological basis, since it does not strictly appear in the context of a factorisation theorem, and therefore it has to be extracted from data. It depends on the order of the perturbation series but is generally assumed to be otherwise universal. Its main effect is to lower the theoretical predictions at large $p_{T}$. Typical parametrisations used in the literature are those by Peterson et al. [43] depending on one parameter $\varepsilon$ and by Kartvelishvili et al. [44] depending on one parameter $\alpha_{K}$.

For the HERA measurements, the fragmentation functions and their uncertainties are considered and accounted for in the original publications $[15,16]$. The measurements of LHCb are provided as hadron-production cross sections and the fragmentation functions have to be applied explicitly in order to use these data in the QCD analysis. In addition, fragmentation fractions describing the probability of a quark to fragment into a particular hadron have to be applied. The fragmentation fractions for $c$-flavoured hadrons are taken from [45] and for $b$-flavoured hadrons from [14].

So far, no fragmentation measurements were performed in $p p$ collisions. Because of similarities of the $c$-quarkproduction kinematics at HERA and LHCb, the Kartvelishvili fragmentation function [44] with $\alpha_{K}=4.4 \pm 1.7$, as obtained from corresponding HERA measurements [46,47] extracted for the NLO FFNS scheme, is applied for predictions of the $\mathrm{LHCb}$ measurements of charm-hadron production. The fragmentation is performed in the laboratory frame by rescaling the quark three-momentum, with the energy of the produced hadron being calculated using the hadron mass. This procedure is used for $D^{+}$and $D_{s}^{+}$mesons, and for $\Lambda_{c}^{+}$ baryons. For $D^{0}$ - and $D^{+}$-meson production, the contribution from $D^{*+}$ and $D^{* 0}$ mesons is treated as described in [48]. For beauty production, the value $\alpha_{K}=11 \pm 4$ is used for all $b$-flavoured hadrons, corresponding to measurements at LEP [49].

The fragmentation-fraction uncertainties are assigned to the measurements and are treated as correlated, while the uncertainties arising from the variations of assumptions on the fragmentation functions are treated in the form of variations of the theory predictions in the QCD fit.

\section{Details of the QCD analysis}

The open source QCD fit framework for PDF determination HERAFitter [24], version 1.0.0, is used. The partons are evolved by using the QCDNUM program [50]. In the presented study, collinear factorisation is assumed throughout the analysis performed using the DGLAP [1-9] evolution equations. With the available techniques, no direct tests of non-DGLAP models are possible with the data used here. Such tests would require significant developments of the theory and of the corresponding QCD analysis tools, which is beyond the scope of this paper.

The analysis-specific modifications to HERAFitter address the heavy-flavour treatment as follows. The massive FFNS [51-54] with the number of flavours $N_{f}=3$ is used for the treatment of heavy-flavour contributions. The calculation of one-particle inclusive heavy-quark-production cross sections in hadron collisions at NLO according to [27] is implemented by using original routines from the MNR code [55]. The results agree with those obtained with the original MNR code at a level of accuracy better than $1 \%$.

The 3-flavour strong coupling constant in the NLO $\overline{\mathrm{MS}}$ scheme is set to $\alpha_{S}\left(m_{Z}\right)^{N_{f}=3}=0.1059 \pm 0.0005$, which corresponds to the world average value of $\alpha_{S}\left(m_{Z}\right)^{N_{f}=5}=$ $0.1185 \pm 0.0006$, using two-loop evolution equations [50].

The $Q^{2}$ range of the inclusive HERA data is restricted to $Q^{2}>Q_{\min }^{2}=3.5 \mathrm{GeV}^{2}$. The procedure for the determination of the PDFs follows the approach used in the HERAPDF1.0 QCD fit [10]. The following independent combinations of parton distributions are chosen in the fit procedure at the initial scale of the QCD evolution $Q_{0}^{2}=1.4 \mathrm{GeV}^{2}$ : the valencequark distributions $x u_{\mathrm{v}}(x), x d_{\mathrm{v}}(x)$, the gluon distribution $x g(x)$ and the $u$-type and $d$-type anti-quark distributions (which are identical to the sea-quark distributions), $x \overline{\mathrm{U}}(x)$, $x \overline{\mathrm{D}}(x)$, where $x \overline{\mathrm{U}}(x)=x \bar{u}(x)$ and $x \overline{\mathrm{D}}(x)=x \bar{d}(x)+x \bar{s}(x)$. At the scale $Q_{0}$, the parton distributions are represented by

$$
\begin{aligned}
& x u_{\mathrm{v}}(x)=A_{u_{\mathrm{v}}} x^{B_{u_{\mathrm{v}}}}(1-x)^{C_{u_{\mathrm{v}}}}\left(1+E_{u_{\mathrm{v}}} x^{2}\right), \\
& x d_{\mathrm{v}}(x)=A_{d_{\mathrm{v}}} x^{B_{d_{\mathrm{v}}}}(1-x)^{C_{d_{\mathrm{v}}}}, \\
& x \overline{\mathrm{U}}(x)=A_{\overline{\mathrm{U}}} x^{B_{\overline{\mathrm{U}}}}(1-x)^{C_{\overline{\mathrm{U}}}}, \\
& x \overline{\mathrm{D}}(x)=A_{\overline{\mathrm{D}}} x^{B_{\overline{\mathrm{D}}}}(1-x)^{C_{\overline{\mathrm{D}}},} \\
& x g(x)=A_{g} x^{B_{g}}(1-x)^{C_{g}}-A_{g}^{\prime} x^{B_{g}^{\prime}}(1-x)^{C_{g}^{\prime}} .
\end{aligned}
$$


The normalisation parameters $A_{u_{\mathrm{v}}}, A_{d_{\mathrm{v}}}, A_{g}$ are determined by the QCD sum rules, the $B$ parameters are responsible for the small- $x$ behaviour of the PDFs, and the parameters $C$ describe the shape of the distribution as $x \rightarrow 1$. A flexible form for the gluon distribution is adopted with the choice of $C_{g}^{\prime}=25$ motivated by the approach of the MSTW group [56,57]. The $s$-quark distribution is defined through $x$-independent strangeness fraction, $f_{s}$, of the $d$ type sea, $x \bar{s}=f_{s} x \bar{D}$ at $Q_{0}^{2}$, where $f_{s}=0.31_{-0.08}^{+0.19}$ as in the analysis of [57], including the recent complementary measurement [58]. Additional constraints $B_{\overline{\mathrm{U}}}=B_{\overline{\mathrm{D}}}$ and $A_{\overline{\mathrm{U}}}=A_{\overline{\mathrm{D}}}\left(1-f_{s}\right)$ are imposed, with $x \bar{u} \rightarrow x \bar{d}$ as $x \rightarrow 0$. The analysis is performed by fitting the remaining 13 free parameters in Eqs. (1-5).

The PDF parameters are determined in HERAFitter by minimisation of a $\chi^{2}$-function taking into account correlated and uncorrelated uncertainties [24] of the measurements. Systematic uncertainties are assumed to be proportional to the central prediction values, whereas statistical uncertainties scale with the square root of the predictions. Correlated uncertainties are treated using a nuisance-parameter representation [24]. To minimise biases arising from the likelihood transition to $\chi^{2}$ when the scaling of the errors is applied, a logarithmic correction is added to the $\chi^{2}$-function [59].

The heavy-quark masses are left free in the fit. They are well constrained by the measurements of charm and beauty production in DIS and the fitted values (see Table 2 in the Appendix A) are consistent with the ones obtained in the corresponding HERA analyses $[15,16]$ within the intrinsic

\section{PROSA}

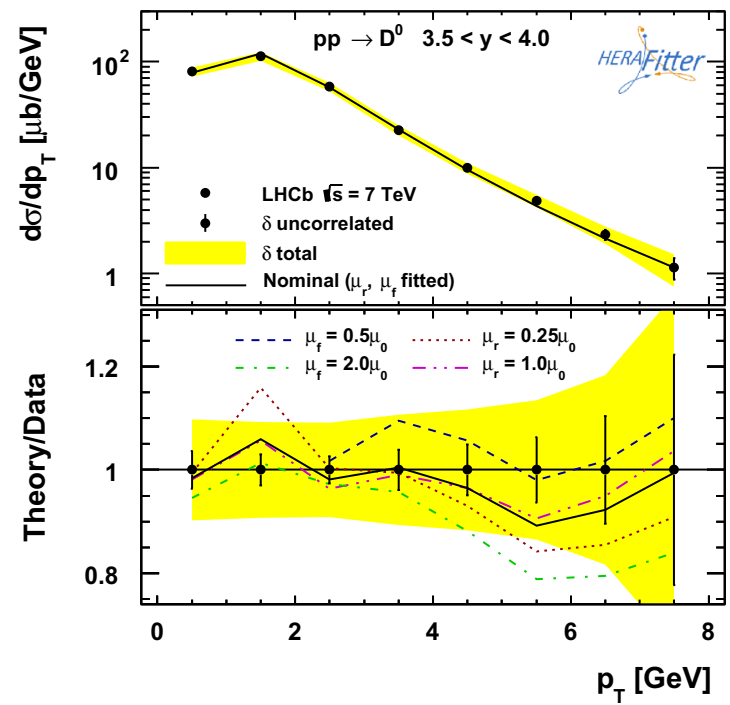

Fig. 2 Data to theory comparison for a representative subset of the $\mathrm{LHCb}$ absolute cross sections for the production of $D^{0}$ mesons for $3.5<y<4.0$ (left) and of $B^{+}$mesons for $3.0<y<3.5$ (right). In the bottom panels the ratios theory/data for the nominal variant of the fit and the scale variations are shown. For demonstration purposes, the theoretical systematic uncertainty of the pole-mass definition [60-62].

The QCD analysis is performed twice using either absolute or normalised differential cross sections of heavy-flavour production from $\mathrm{LHCb}$ measurements, as defined in Sect. 2. The implementation of the theory calculations [63] as described in Sect. 3 allows the pQCD scales, i.e. the parameters $A_{r, f}^{c, b}$, and the values for the pole mass of the heavy quarks to be changed at each fit iteration.

In the QCD analysis using the normalised $\mathrm{LHCb}$ cross sections, the pQCD scales are fixed to $A_{r}=A_{f}=1$ for the central result. The scale dependence is studied by varying the pQCD scales independently such that $0.5 \leq A_{r}, A_{f} \leq 2$. $A^{c}$ and $A^{b}$ are always varied simultaneously. The resulting scale dependence is small, since it is largely absorbed by the normalisation, as illustrated in Appendix A.

In the variant of the fit using the absolute $\mathrm{LHCb}$ cross sections, the scale dependence of the predicted cross section is the dominant theoretical uncertainty. The same scale-choice and variation procedure, as applied for the variant of the fit using the normalised LHCb cross sections, leads to unacceptably high $\chi^{2}$ values of the respective fits [63]. Therefore, the four scales technically are treated as independent fully correlated systematic uncertainties for the central result. Since the pQCD scales are not physical parameters, the related uncertainties are not obtained from the fit. Instead, the effect of the scale choice on the other fitted parameters is evaluated by an independent variation of $A_{f}$ in the range $0.5<A_{f}^{c}=$

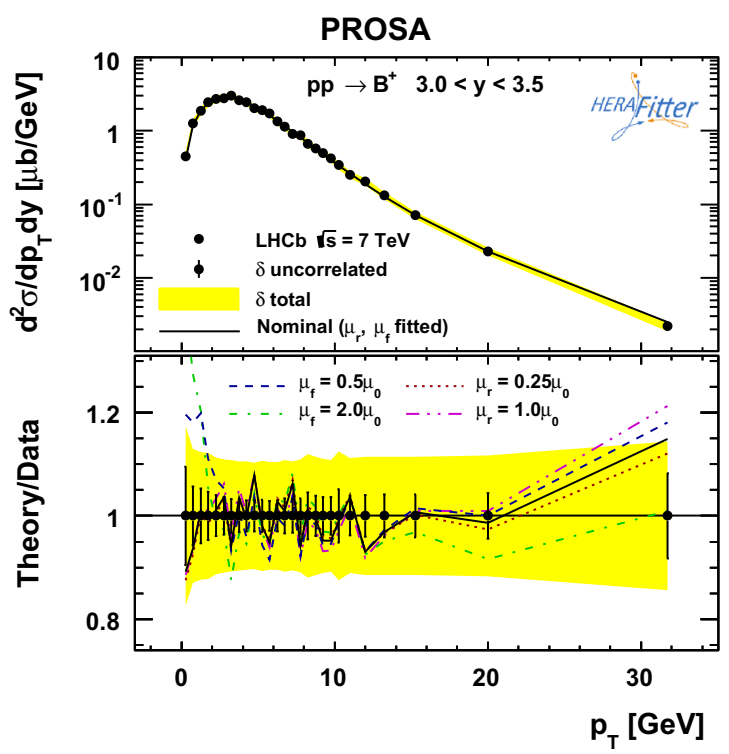

correlated shifts for the data points obtained in the fit using nuisance parameters are applied to the theoretical predictions. The uncorrelated uncertainties for the data points are shown as they are rescaled in the fit, while the total uncertainties are shown as not rescaled 


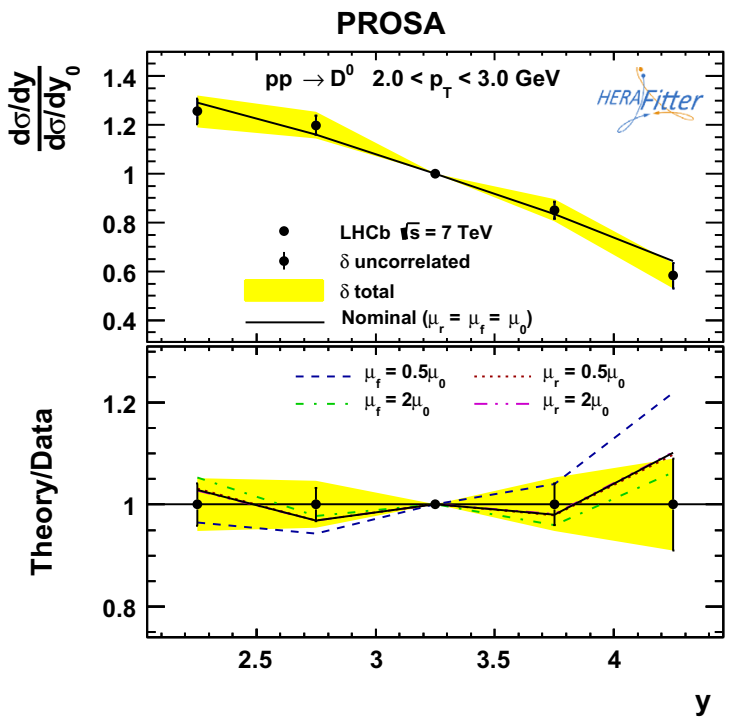

Fig. 3 Data to theory comparison for a representative subset of the LHCb normalised cross sections for the production of $D^{0}$ mesons for $2.0<p_{T}<3.0 \mathrm{GeV}$ (left) and of $B^{+}$mesons for $3.0<p_{T}<3.5 \mathrm{GeV}$ (right). The central rapidity bins are fixed to 1 by the definition of the normalised cross sections. In the bottom panels the ratios theory/data for the nominal variant of the fit and the scale variations are shown.

$A_{f}^{b}<2$ with $A_{r}^{c}$ and $A_{r}^{b}$ as free parameters, or $A_{r}$ in the range $0.25<A_{r}^{c}=A_{r}^{b}<1$ with $A_{f}^{c}$ and $A_{f}^{b}$ being free parameters. For the variation $A_{f}^{c}=A_{f}^{b}=0.5$, a cut $p_{T}>2$ $\mathrm{GeV}$ is applied for the charm $\mathrm{LHCb}$ data to ensure that the factorisation scale is above $1 \mathrm{GeV}^{2}$, since this is technically required in the QCDNUM program. This procedure ensures an acceptable fit quality for all variations [63], as required for a meaningful extraction of the other uncertainties. Because of the unconventional scale treatment the fit using absolute cross sections is considered to be a cross check.

\section{PDF uncertainties}

The PDF uncertainties are estimated following the approach of HERAPDF1.0 [10] in which experimental, model, and parametrisation uncertainties are taken into account. Experimental uncertainties are evaluated using the Hessian method [24]. A tolerance criterion of $\Delta \chi^{2}=1$ is adopted for defining the fit uncertainties that originate from the experimental uncertainties of the measurements included in the analysis.

Model uncertainties arise from the variations in the values assumed for $Q_{\min }^{2}$ imposed on the HERA data, which is varied in the interval $2.5 \leq Q_{\min }^{2}<5.0 \mathrm{GeV}^{2}$; the fraction of strange quarks, varied in the range $0.23<f_{s}<0.50$ and the value of the strong coupling, varied in the range $0.1054<\alpha_{S}\left(m_{Z}\right)^{N_{F}=3}<0.1064$. The pQCD scales for heavy-quark production in DIS are varied simultaneously by a factor of 2 up and down for both, charm and beauty. For

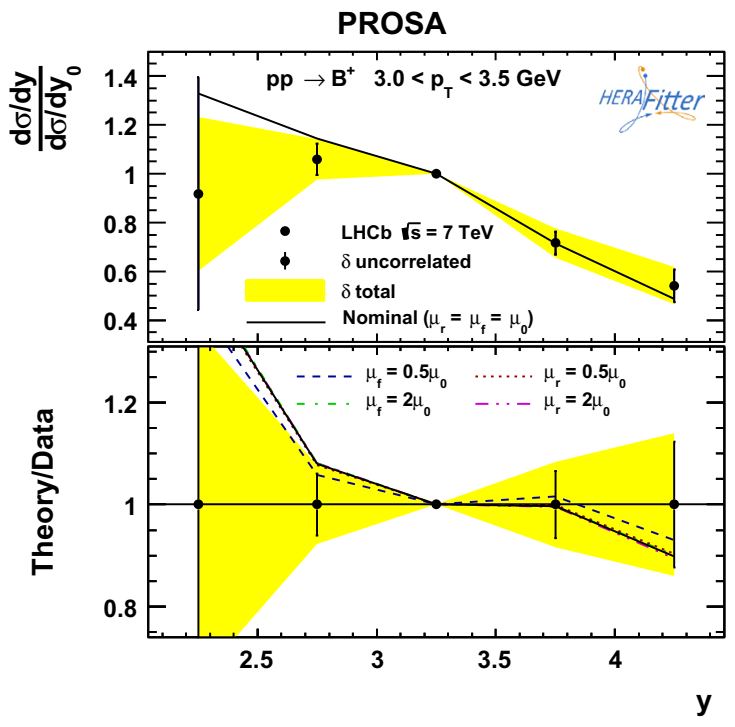

For demonstration purposes, the correlated shifts for the data points obtained in the fit using nuisance parameters are applied to the theoretical predictions. The uncorrelated uncertainties for the data points are shown as they are rescaled in the fit, while the total uncertainties are shown as not rescaled

Table 1 The global and partial $\chi^{2}$ values for the data sets used in the analysis of HERA and LHCb measurements

\begin{tabular}{lll}
\hline Representation of LHCb data & Absolute & Normalised \\
\hline Global $\chi^{2} / n_{\text {dof }}$ & $1073 / 1087$ & $958 / 994$ \\
Global $\chi^{2} p$-value & 0.61 & 0.79 \\
Correlated uncertainties $\chi^{2}$ & 73 & 49 \\
Logarithmic correction $\chi^{2}$ & -129 & 48 \\
Data set & \multicolumn{2}{c}{ Partial $\chi^{2} / n_{\text {dof }}$} \\
NC DIS HERA I comb. $e^{-} p$ & $108 / 145$ & $108 / 145$ \\
NC DIS HERA I comb. $e^{+} p$ & $419 / 379$ & $419 / 379$ \\
CC DIS HERA I comb. $e^{-} p$ & $26 / 34$ & $26 / 34$ \\
CC DIS HERA I comb. $e^{+} p$ & $39 / 34$ & $41 / 34$ \\
$c \bar{c}$ DIS HERA comb. & $78 / 52$ & $47 / 52$ \\
$b \bar{b}$ DIS ZEUS Vertex & $16 / 17$ & $12 / 17$ \\
LHCb $D^{0}$ & $68 / 38$ & $17 / 30$ \\
LHCb $D^{+}$ & $53 / 37$ & $18 / 29$ \\
LHCb $D^{*+}$ & $50 / 31$ & $19 / 22$ \\
LHCb $D_{s}^{+}$ & $24 / 28$ & $11 / 20$ \\
LHCb $\Lambda_{c}^{+}$ & $5.3 / 6$ & $4.9 / 3$ \\
LHCb $B^{+}$ & $99 / 135$ & $81 / 108$ \\
LHCb $B^{0}$ & $66 / 95$ & $35 / 76$ \\
LHCb $B_{s}^{0}$ & $78 / 75$ & $23 / 60$ \\
\hline
\end{tabular}

the fits with the LHCb data, the model uncertainties include theoretical uncertainties for the cross section predictions for heavy-flavoured hadron production, arising from variation of the pQCD scales and of the fragmentation parameters, as 

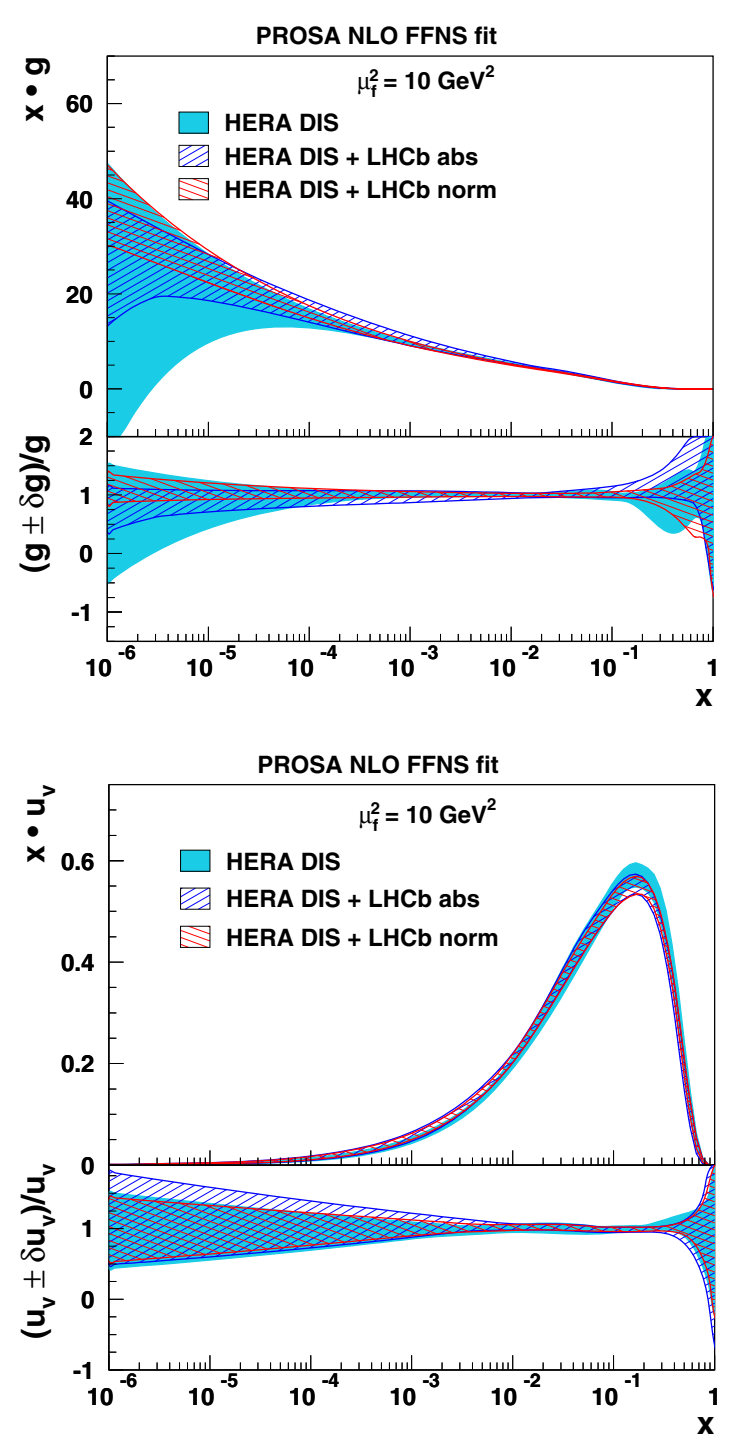

Fig. 4 The gluon (top left), the sea-quark (top right), the $u$-valence quark (bottom left) and the $d$-valence quark (bottom right) distributions represented at $\mu_{f}^{2}=10 \mathrm{GeV}^{2}$, as obtained in the QCD analysis of the HERA-only data (light shaded band) and HERA and LHCb mea-

described in Sect. 3. Uncertainties, arising from these model variations are referred to as MNR uncertainties in the following.

The parametrisation uncertainty is estimated similarly to the HERAPDF1.0 procedure: for all PDFs, additional parameters are added one by one in the functional form of the parametrisations in Eqs. (1-5), in a similar way as described in $[10,15,16]$. Furthermore, the starting scale is varied to $Q_{0}^{2}=1.9 \mathrm{GeV}^{2}$. The parametrisation uncertainty is constructed as an envelope built from the maximal differences between the PDFs resulting from all the parametrisation variations and the central fit at each $x$ value. The total PDF uncertainty is obtained by adding experimental, model and parametrisation uncertainties in quadrature.
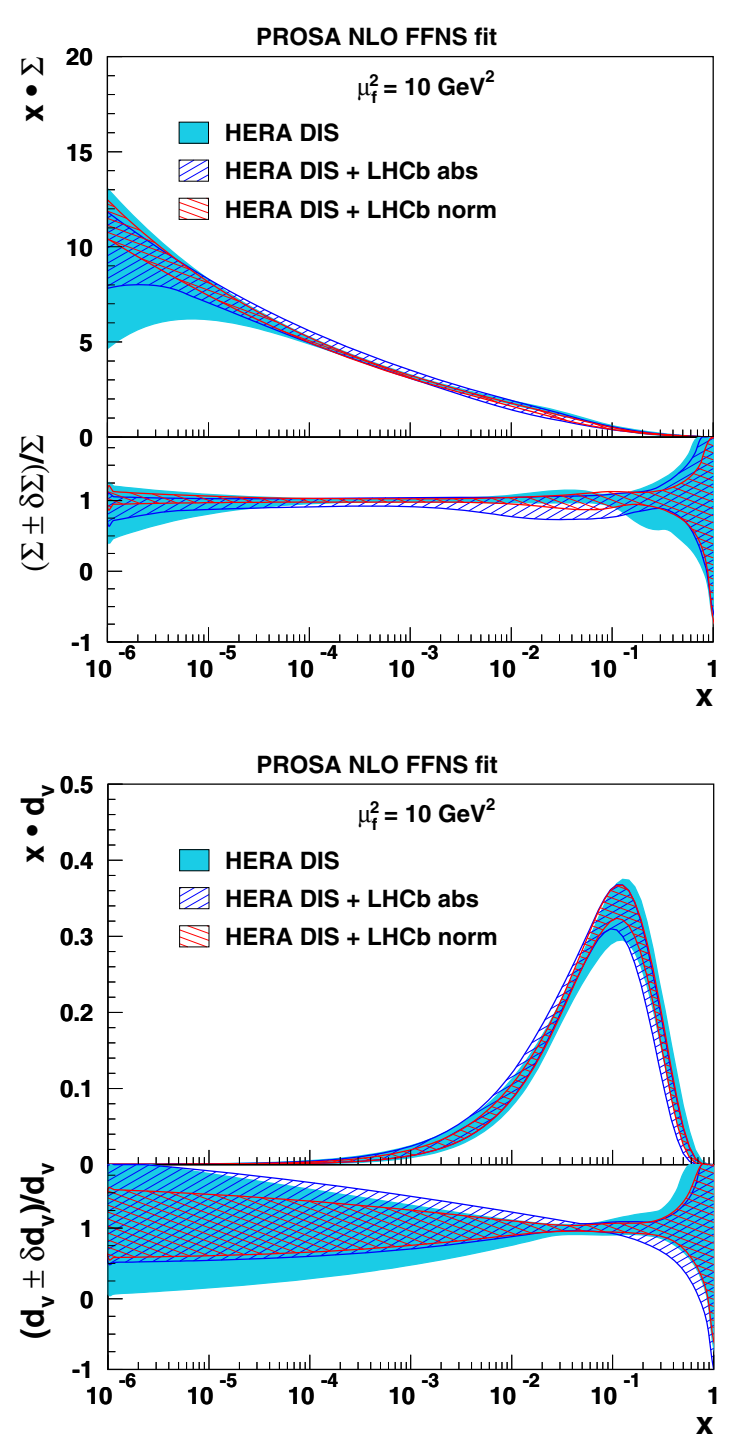

surements and their relevant uncertainties. The sea-quark distribution is defined as $\Sigma=2 \cdot(\bar{u}+\bar{d}+\bar{s})$. The results of the fit using absolute or normalised LHCb cross sections are shown by different hatches. The widths of the bands represent the total uncertainties

\section{Results}

In Fig. 2, the absolute cross sections for $D^{0}$ - and $B^{+}$-meson production in $p p$ collisions are shown for one representative rapidity bin and are compared to the theory predictions as used in the QCD analysis. A significant scale dependence is observed. The normalised cross sections for a representative $p_{T}$ bin of the same data set are compared to the respective theory predictions in Fig. 3. The advantage of using the normalised cross section is a significant reduction of the scale dependence of the theoretical prediction, retaining the sensitivity of the cross sections to the gluon distribution. The reduction of the uncertainty due to the scale variations is related to the fact that the scale choice affects mostly the 

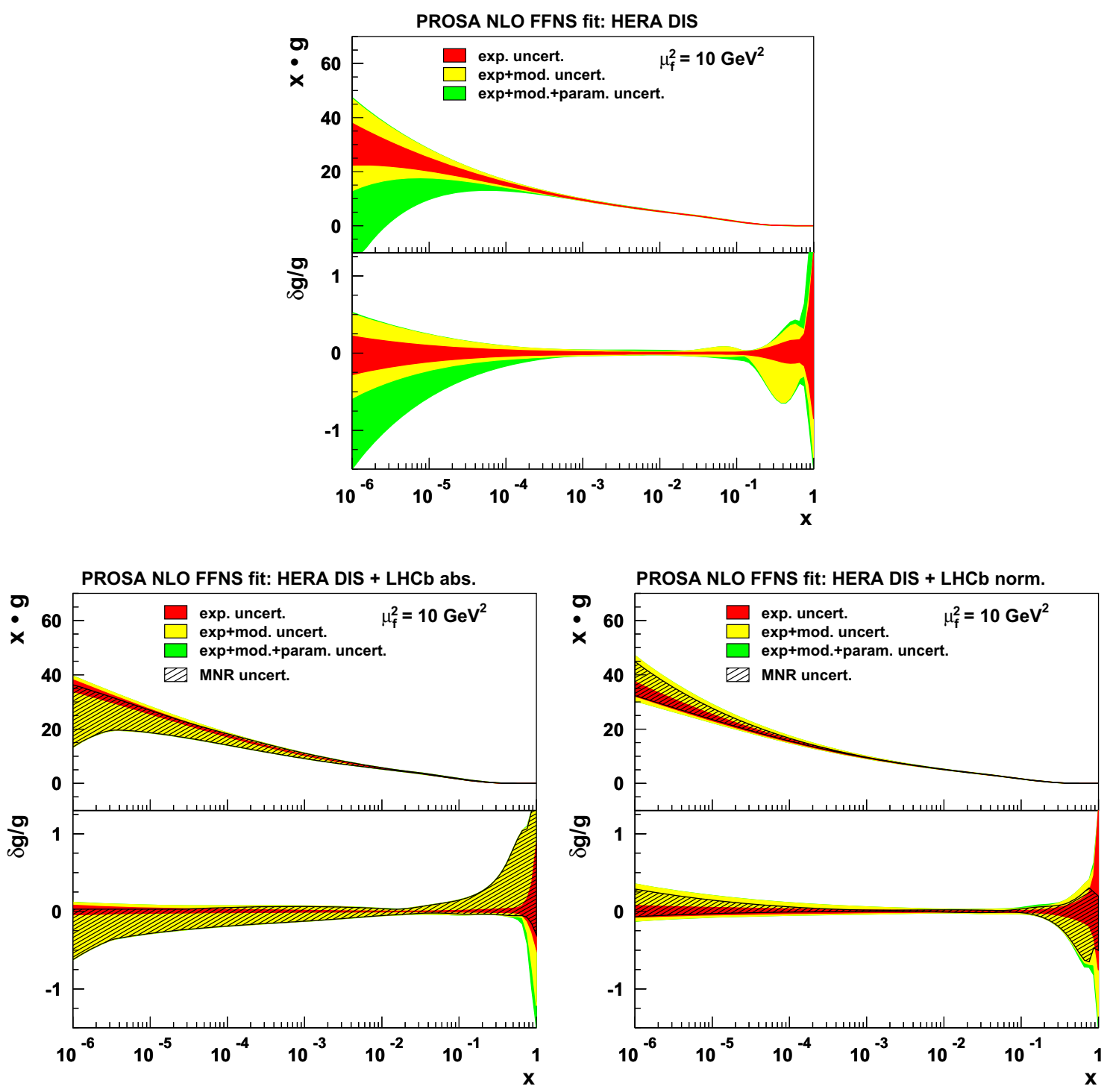

Fig. 5 Individual contributions to the PDF uncertainties on the gluon distributions, obtained in QCD analyses using HERA-only (upper panel), HERA+LHCb absolute (lower panel left) and HERA+LHCb normalised (lower panel right) cross sections of heavy-flavour production

normalisation but only to some extent the shape of heavyquark-production kinematics, as demonstrated in Figs. 6, 7 in the Appendix A.

The fit quality, represented by the total and partial values of $\chi^{2}$ divided by the number of degrees of freedom, $n_{\mathrm{dof}}$, for both variants of the QCD analysis is presented in Table 1 . When the normalised $\mathrm{LHCb}$ cross sections are used in the QCD analysis, $n_{\text {dof }}$ is appropriately reduced for the respective data sets. The fitted parameters are presented in Table 2 in Appendix A.

The resulting gluon, valence-quark and sea-quark distributions with their total uncertainties are presented at $\mu_{f}^{2}=10$ $\mathrm{GeV}^{2}$ in Fig. 4 and compared to the result of the fit, based on solely HERA measurements of inclusive and heavy-flavour DIS. The uncertainties on the gluon and sea-quark distribu- tions at low $x$ are significantly reduced in both cases, using $\mathrm{LHCb}$ absolute or normalised heavy-quark-production cross sections.

In case of the variant of the fit based on normalised $\mathrm{LHCb}$ cross sections, the uncertainties are reduced by more than a factor of three at $x \sim 5 \times 10^{-6}$, which is the edge of the sensitivity of the included measurements (Fig. 1). Consistent results are obtained in the fit using the absolute cross sections, which is considered an important cross check of the selfconsistency of the NLO theory description.

The individual contributions of the experimental, model and parametrisation uncertainties for both cases of using the LHCb measurements are shown in Fig. 5 and compared to the result of the fit using only HERA data. The gluon distribution at low $x$ is constrained by the HERA measurements mostly 
via the sum rules and this results in large parametrisation uncertainties. Once the LHCb measurements are included in the QCD analysis, the gluon distribution is directly probed and the parametrisation dependence of the PDF is significantly reduced.

The main differences in the PDF uncertainties between the fits using the absolute and normalised LHCb cross sections are caused by the MNR uncertainties. The variation of the pQCD scales in the prediction of the absolute cross section of heavy-flavour production in $p p$ collisions leads to significant changes in the normalisation of the cross section and represents the dominant uncertainty on the PDFs. The variations of the assumptions on the fragmentation parameters result in a negligible uncertainty as compared to those due to the scale variations, see e.g. Fig. 7.13 [63], since changes of the $p_{T}$ shape due to variations of the fragmentation function can be compensated by small changes in the scales.

In the case of the PDF fit using the normalised $\mathrm{LHCb}$ cross sections, the MNR uncertainty is strongly reduced, since variations of pQCD scales and of the fragmentation parameters do not significantly affect the shape of the $y$ distribution for heavy-flavour production. Therefore this is considered to be the primary result of this paper, while the consistency between the absolute and normalised variants is considered to be an important cross check.

\section{Conclusions}

The sensitivity of heavy-flavour production in $p p$ collisions to the low- $x$ gluon distribution was studied in a comprehensive QCD analysis at NLO. The measurements of $c$ - and $b$-hadron-production cross sections at the $\mathrm{LHCb}$ experiment are included into a PDF fit together with inclusive and heavyflavour-production measurements in DIS at HERA. Since the bulk of the heavy-flavour data is close to the kinematic threshold, the fixed-flavour number scheme at NLO order is used for the predictions of heavy-flavour production in $e p$ and $p p$ collisions. A significant reduction of the parametrisation uncertainty of the gluon distribution at very low $x$ is observed, as compared to the result of the PDF fit using only HERA DIS data.

Two ways of using the $\mathrm{LHCb}$ measurements in the fit are studied. Although the absolute differential cross-section measurements contain more information, the resulting PDFs suffer from large theoretical uncertainty due to uncalculated higher-order corrections, estimated by the variation of the pQCD scales. By using only the rapidity shape information in the normalised cross sections for the final result, this uncertainty is significantly reduced for the PDF extraction.

The present analysis has illustrated the high potential of the $\mathrm{LHCb}$ measurements to constrain the gluon distribution at low $x$, and global PDF fits clearly can profit from the inclusion of such data. Precise measurements of normalised cross sections of heavy-flavour production in the forward kinematic range of the LHC therefore have a great potential to further improve the constraints on the PDFs.

In order to fully exploit the additional constraints from absolute LHC charm and beauty cross sections, a significant reduction of the theoretical uncertainties, e.g. through threshold resummation and/or (partial) NNLO calculations with codes suitable for a usage in QCD analyses, is desirable.

Acknowledgments This work has been supported in part by the Helmholtz Association under contract numbers HGF-SO-072 and VHHA-101. Supported in part by the EU-TMR Network Higgstools.

Open Access This article is distributed under the terms of the Creative Commons Attribution 4.0 International License (http://creativecomm ons.org/licenses/by/4.0/), which permits unrestricted use, distribution, and reproduction in any medium, provided you give appropriate credit to the original author(s) and the source, provide a link to the Creative Commons license, and indicate if changes were made. Funded by SCOAP ${ }^{3}$.

\section{Appendix A}

See Table 2 and Figs. 6 and 7.

Table 2 The fitted parameters for the NLO QCD analysis using HERA and LHCb measurements. The value of strong coupling $\alpha_{S}\left(m_{Z}\right)^{N_{f}=3}=$ 0.1059 is used (which corresponds to $\alpha_{S}\left(m_{Z}\right)^{N_{f}=5}=0.1185$ ). The listed uncertainties correspond to those associated to the experimental measurements used in the fit. Uncertainties are not quoted for parameters that are fixed. The correlation matrix can be made available upon request

\begin{tabular}{lll}
\hline Parameter & Absolute & Normalised \\
\hline$B_{g}$ & $-0.14 \pm 0.07$ & $-0.08 \pm 0.10$ \\
$C_{g}$ & $6.83 \pm 0.31$ & $5.23 \pm 0.34$ \\
$A_{g}^{\prime}$ & $1.74 \pm 0.22$ & $1.29 \pm 0.32$ \\
$B_{g}^{\prime}$ & $-0.19 \pm 0.04$ & $-0.16 \pm 0.05$ \\
$B_{u_{\mathrm{v}}}$ & $0.668 \pm 0.020$ & $0.649 \pm 0.021$ \\
$C_{u_{\mathrm{v}}}$ & $4.99 \pm 0.23$ & $4.98 \pm 0.23$ \\
$E_{u_{\mathrm{v}}}$ & $12.2 \pm 2.4$ & $13.5 \pm 2.7$ \\
$B_{d_{\mathrm{v}}}$ & $0.93 \pm 0.09$ & $0.96 \pm 0.09$ \\
$C_{d_{\mathrm{v}}}$ & $5.50 \pm 0.56$ & $5.59 \pm 0.55$ \\
$C_{\overline{\mathrm{U}}}$ & $1.63 \pm 0.21$ & $1.63 \pm 0.24$ \\
$A_{\overline{\mathrm{D}}}$ & $0.173 \pm 0.007$ & $0.158 \pm 0.007$ \\
$B_{\overline{\mathrm{D}}}$ & $-0.146 \pm 0.006$ & $-0.155 \pm 0.007$ \\
$C_{\overline{\mathrm{D}}}$ & $10.4 \pm 2.5$ & $15.1 \pm 4.2$ \\
$m_{c}[\mathrm{GeV}]$ & $1.709 \pm 0.024$ & $1.257 \pm 0.014$ \\
$m_{b}[\mathrm{GeV}]$ & $4.67 \pm 0.08$ & $4.19 \pm 0.13$ \\
$A_{f}^{c}$ & $0.659 \pm 0.020$ & 1.0 \\
$A_{f}^{b}$ & $0.262 \pm 0.007$ & 1.0 \\
$A_{r}^{c}$ & $0.444 \pm 0.021$ & 1.0 \\
$A_{r}^{b}$ & $0.335 \pm 0.024$ & 1.0 \\
\hline & &
\end{tabular}


PROSA

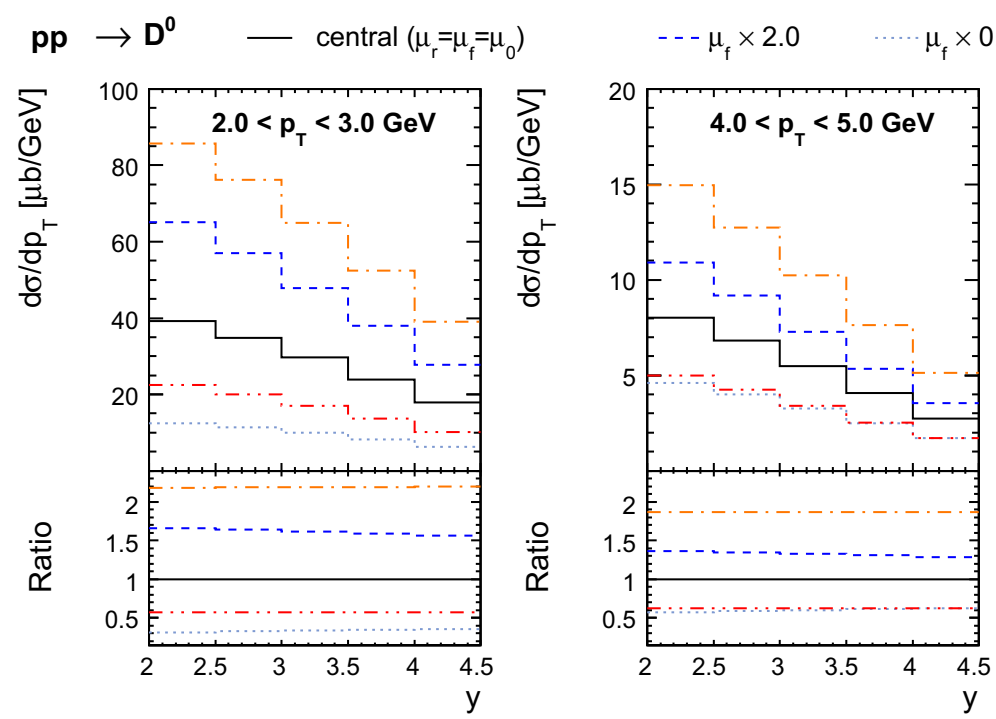
$\times 0.5$
$-\cdots \mu_{\mathrm{r}} \times 2.0$
$-\mu_{r} \times 0.5$
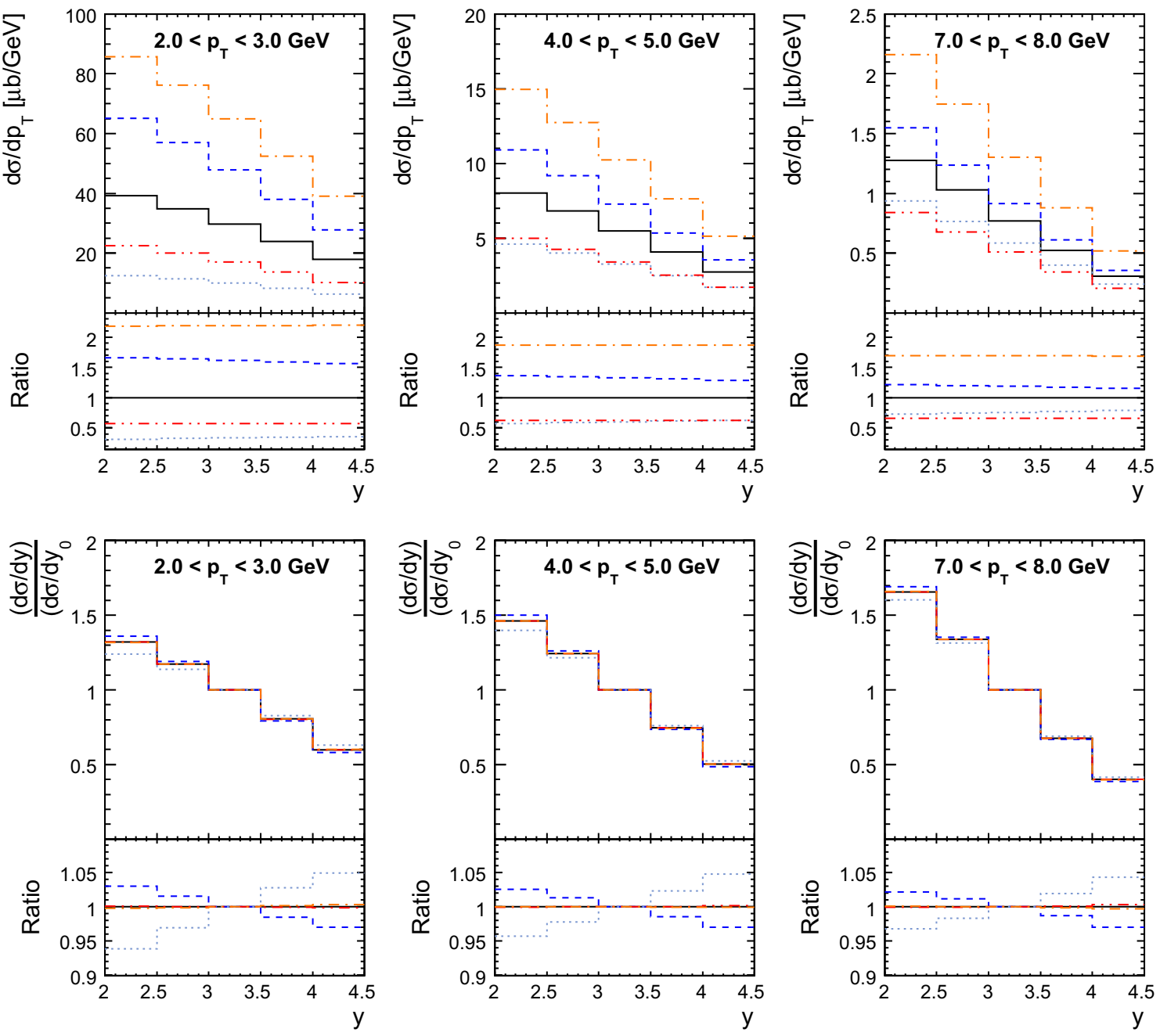

Fig. 6 NLOQCD predictions for charm LHCb data with different scale choices for absolute (top) and normalised (bottom) cross sections. The choice. The predictions are obtained by using the FFNS variant of the lower panels indicate the ratio of the predictions to the central scale

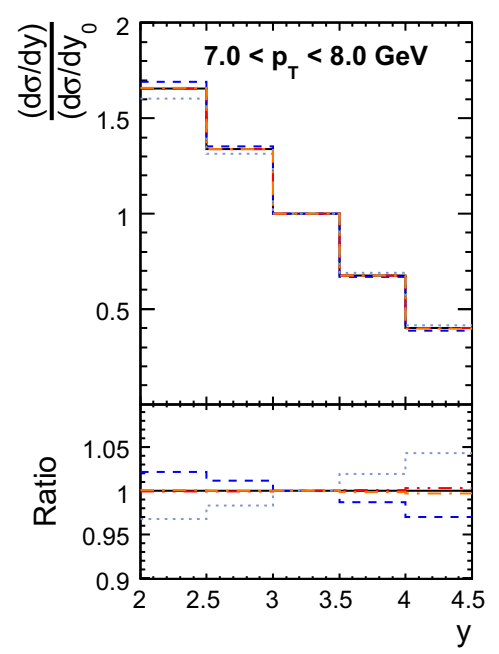

MSTW 2008 PDFs [64] with $N_{f}=3$; the charm mass is set to $m_{c}=1.5$ $\mathrm{GeV}$ 


\section{PROSA}

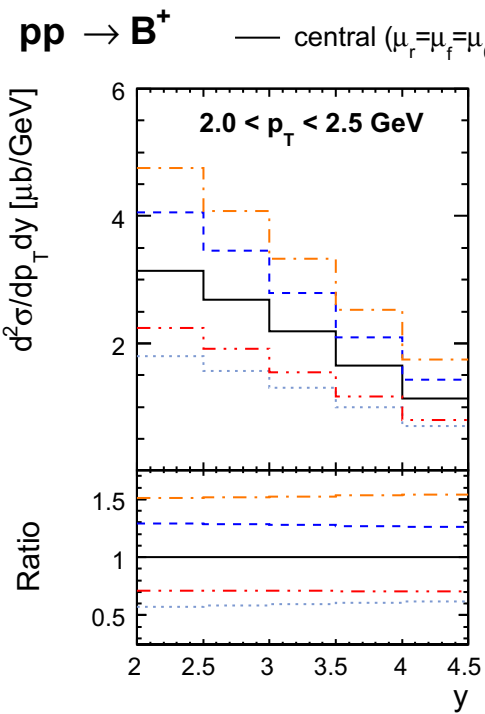

- - $\mu_{f} \times 2.0$

$\mu_{f} \times 0.5$

$-\cdots \mu_{r} \times 2.0$

$\mu_{r} \times 0.5$
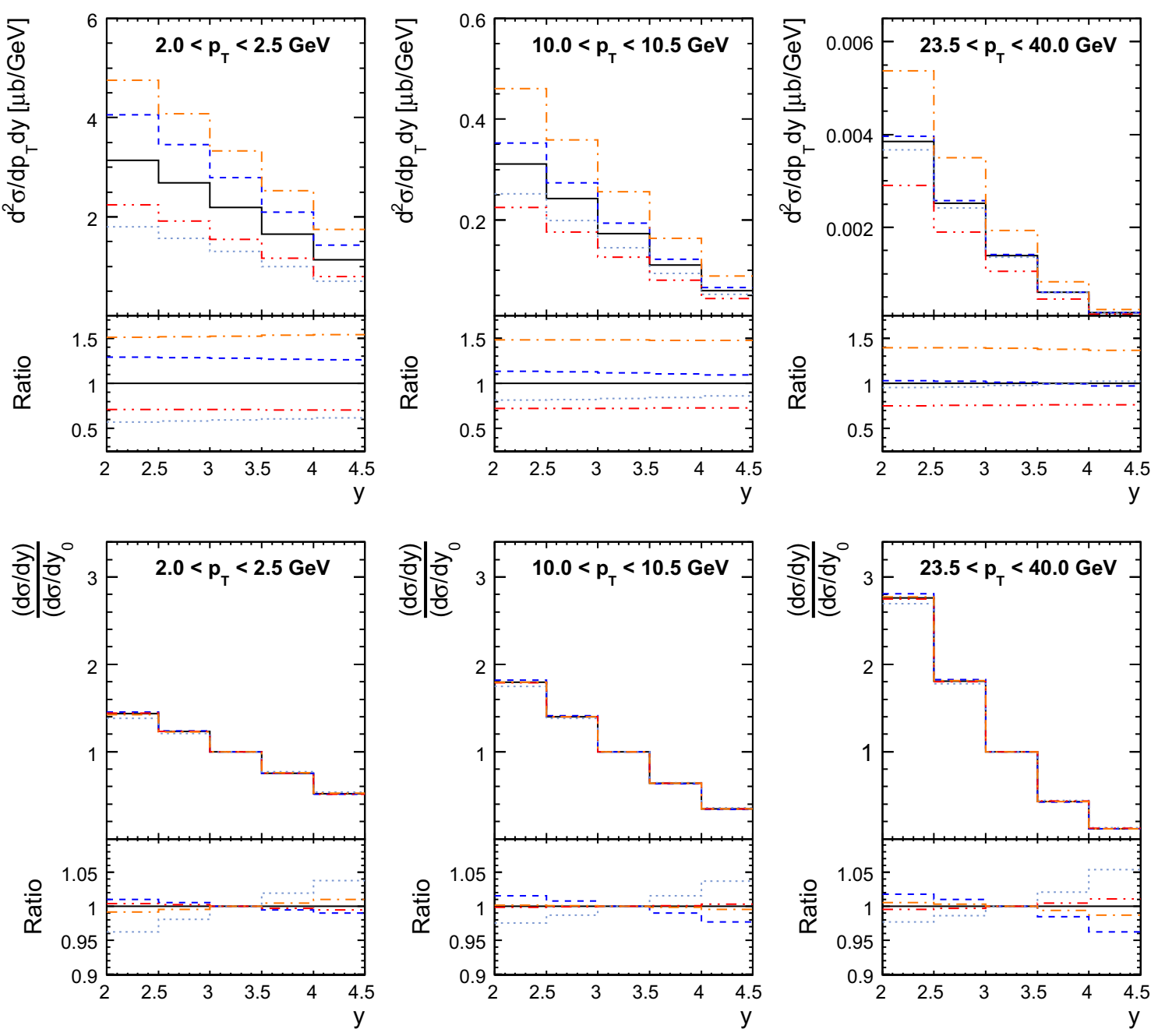

Fig. 7 NLO QCD predictions for beauty LHCb data with different scale choices for absolute (top) and normalised (bottom) cross sections. The lower panels indicate the ratio of the predictions to the central scale choice. The predictions are obtained using the FFNS variant of the MSTW 2008 PDFs [64] with $N_{f}=3$; the beauty mass is set to $m_{b}=4.5 \mathrm{GeV}$

\section{References}

1. D.J. Gross, F. Wilczek, Phys. Rev. D 8, 3633 (1973). doi:10.1103/ PhysRevD.8.3633

2. D.J. Gross, F. Wilczek, Phys. Rev. D 9, 980 (1974). doi:10.1103/ PhysRevD.9.980

3. H. Georgi, H.D. Politzer, Phys. Rev. D 9, 416 (1974). doi:10.1103/ PhysRevD.9.416

4. V.N. Gribov, L.N. Lipatov, Sov. J. Nucl. Phys. 15, 438 (1972)

5. G. Altarelli, G. Parisi, Nucl. Phys. B 126, 298 (1977). doi:10.1016/ 0550-3213(77)90384-4

6. G. Curci, W. Furmanski, R. Petronzio, Nucl. Phys. B 175, 27 (1980). doi:10.1016/0550-3213(80)90003-6

7. W. Furmanski, R. Petronzio, Phys. Lett. B 97, 437 (1980). doi:10. 1016/0370-2693(80)90636-X

8. S. Moch, J.A.M. Vermaseren, A. Vogt, Nucl. Phys. B 688, 101 (2004). arXiv:hep-ph/0403192. doi:10.1016/j.nuclphysb.2004.03. 030
9. A. Vogt, S. Moch, J.A.M. Vermaseren, Nucl. Phys. B 691, 129 (2004). arXiv:hep-ph/0404111. doi:10.1016/j.nuclphysb.2004.04. 024

10. F.D. Aaron et al., H1 and ZEUS Collaboration. JHEP 1001, 109 (2010). arXiv:0911.0884. doi:10.1007/JHEP01(2010)109

11. A. Cooper-Sarkar, P. Mertsch, S. Sarkar, JHEP 1108, (2011) 042 arXiv:1106.3723. doi:10.1007/JHEP08(2011)042

12. L. Pasquali, M.H. Reno, I. Sarcevic, Phys. Rev. D 59 , 034020 (1999). arXiv:hep-ph/9806428. doi:10.1103/PhysRevD. 59.034020

13. R. Aaij et al., LHCb Collaboration. Nucl. Phys. B 871, 1 (2013). arXiv:1302.2864. doi:10.1016/j.nuclphysb.2013.02.010

14. R. Aaij et al., LHCb Collaboration. JHEP 1308, 117 (2013). arXiv: 1306.3663. doi:10.1007/JHEP08(2013)117

15. F.D. Aaron et al., $\mathrm{H} 1$ and ZEUS Collaborations. Eur. Phys. J. C 73, 2311 (2013). arXiv:1211.1182. doi:10.1140/epjc/ s10052-013-2311-3 
16. H. Abramowicz et al., ZEUS Collaboration. JHEP 1409, 127 (2014). arXiv:1405.6915. doi:10.1007/JHEP09(2014)127

17. H. Kawamura, N.A. Lo, Presti, S. Moch and A. Vogt. Nucl. Phys. B 864, 399 (2012). arXiv:1205.5727. doi:10.1016/j.nuclphysb.2012. 07.001

18. M. Guzzi, K. Lipka and S. O. Moch, JHEP 1501, 082 (2015). arXiv:1406.0386. doi:10.1007/JHEP01(2015)082

19. N. Kidonakis, E. Laenen, S. Moch, R. Vogt, Phys. Rev. D 64, 114001 (2001). arXiv:hep-ph/0105041. doi:10.1103/PhysRevD. 64.114001

20. N. Kidonakis, Phys. Rev. D 91(3), 031501 (2015). arXiv:1411.2633. doi:10.1103/PhysRevD.91.031501

21. S. Alekhin, J. Blümlein, S. Moch, Phys. Rev. D 86, 054009 (2012). arXiv:1202.2281. doi:10.1103/PhysRevD.86.054009

22. I. Bierenbaum, J. Blümlein, S. Klein, Phys. Lett. B 672, 401 (2009). arXiv:0901.0669. doi:10.1016/j.physletb.2009.01.057

23. S. Alekhin, OPENQCDRAD-1.5. http://www-zeuthen.desy.de/ alekhin/OPENQCDRAD

24. S. Alekhin, O. Behnke, P. Belov, S. Borroni, M. Botje, D. Britzger, S. Camarda, A. M. Cooper-Sarkar et al., HERAFitter. arXiv: 1410.4412

25. P. Nason, S. Dawson, R.K. Ellis, Nucl. Phys. B 303, 607 (1988). doi:10.1016/0550-3213(88)90422-1

26. W. Beenakker, H. Kuijf, W.L. van Neerven, J. Smith, Phys. Rev. D 40, 54 (1989). doi:10.1103/PhysRevD.40.54

27. P. Nason, S. Dawson, R.K. Ellis, Nucl. Phys. B 327, 49 (1989). doi:10.1016/0550-3213(89)90286-1

28. M.L. Mangano, P. Nason, G. Ridolfi, Nucl. Phys. B 373, 295 (1992). doi:10.1016/0550-3213(92)90435-E

29. C. Albajar et al., UA1 collaboration. Phys. Lett. B 369, 46 (1996). doi:10.1016/0370-2693(95)01539-6

30. C. Albajar et al., UA1 collaboration. Z. Phys. C 61, 41 (1994). doi:10.1007/BF01641885

31. C. Albajar et al., UA1 collaboration, Phys. Lett. B 256, 121 (1991). Erratum-ibid. B 262, 497 (1991). doi:10.1016/ 0370-2693(91)90228-I

32. A. Abulencia et al., CDF Collaboration. Phys. Rev. D 75, 012010 (2007). arXiv:hep-ex/0612015. doi:10.1103/PhysRevD. 75.012010

33. M. Cacciari, P. Nason, Phys. Rev. Lett. 89, 122003 (2002). arXiv:hep-ph/0204025. doi:10.1103/PhysRevLett.89.122003

34. M. Cacciari, M. Greco, P. Nason, JHEP. 9805, 007 (1998). arXiv:hep-ph/9803400. doi:10.1088/1126-6708/1998/05/007

35. B.A. Kniehl, G. Kramer, I. Schienbein, H. Spiesberger, Phys. Rev. D 77, 014011 (2008). arXiv:0705.4392. doi:10.1103/PhysRevD. 77.014011

36. S. Frixione et al., Phys. Lett. B 348, 633 (1995). doi:10.1016/ 0370-2693(95)00163-F

37. S. Frixione, P. Nason, G. Ridolfi, Nucl. Phys. B 454, 3 (1995). arXiv:hep-ph/9506226. doi:10.1016/0550-3213(95)00445-X

38. S. Aid et al., H1 Collaboration. Nucl. Phys. B 472, 32 (1996). arXiv:hep-ex/9604005. doi:10.1016/0550-3213(96)00275-1

39. M. Derrick et al., ZEUS Collaboration. Phys. Lett. B 349, 225 (1995). arXiv:hep-ex/9502002. doi:10.1016/ 0370-2693(95)00253-H

40. W. Beenakker, W.L. van Neerven, R. Meng, G.A. Schuler, J. Smith, Nucl. Phys. B 351, 507 (1991). doi:10.1016/ S0550-3213(05)80032-X
41. B. A. Kniehl, G. Kramer, I. Schienbein, H. Spiesberger. arXiv:1502.01001

42. B.A. Kniehl, G. Kramer, I. Schienbein, H. Spiesberger, Eur. Phys. J. C 72, 2082 (2012). arXiv:1202.0439. doi:10.1140/epjc/ s10052-012-2082-2

43. C. Peterson, D. Schlatter, I. Schmitt, P. Zerwas, Phys. Rev. D 27, 105 (1983). doi:10.1103/PhysRevD.27.105

44. V. Kartvelishvili, A. Likhoded, V. Petrov, Phys. Lett. B 78, 615 (1978). doi:10.1016/0370-2693(78)90653-6

45. E. Lohrmann. arXiv:1112.3757

46. F. Aaron et al., H1 Collaboration. Eur. Phys. J. C 59, 589 (2009). arXiv:0808.1003. doi:10.1140/epjc/s10052-008-0792-2

47. S. Chekanov et al., ZEUS Collaboration. JHEP 0904, 082 (2009). arXiv:0901.1210. doi:10.1088/1126-6708/2009/04/082

48. M. Cacciari, P. Nason, JHEP. 0309, 006 (2003). arXiv:hep-ph/0306212. doi:10.1088/1126-6708/2003/09/006

49. P. Nason, C. Oleari, Nucl. Phys. B 565, 245 (2000). arXiv:hep-ph/9903541. doi:10.1016/S0550-3213(99)00673-2

50. M. Botje, Comput. Phys. Commun. 182, 490 (2011). arXiv:1005.1481. doi:10.1016/j.cpc.2010.10.020

51. E. Witten, Nucl. Phys. B 104, 445 (1976). doi:10.1016/ 0550-3213(76)90111-5

52. E. Laenen, S. Riemersma, J. Smith, W.L. van Neerven, Nucl. Phys. B 392, 162 (1993). doi:10.1016/0550-3213(93)90201-Y

53. I. Bierenbaum, J. Blümlein, S. Klein, Nucl. Phys. B 820, 417 (2009). arXiv:0904.3563. doi:10.1016/j.nuclphysb.2009.06.005

54. J. Blümlein, A. De Freitas, C. Schneider. arXiv:1411.5669

55. The MNR code. http://www.ge.infn.it/ ridolfi/hvqlibx.tgz

56. R.S. Thorne, Phys. Rev. D 73, 054019 (2006). arXiv:hep-ph/0601245. doi:10.1103/PhysRevD.73.054019

57. A.D. Martin, W.J. Stirling, R.S. Thorne, G. Watt, Eur. Phys. J. C 63, 189 (2009). arXiv:0901.0002. doi:10.1140/epjc/ s10052-009-1072-5

58. G. Aad et al., ATLAS Collaboration. Phys. Rev. Lett. 109, 012001 (2012). arXiv:1203.4051.doi:10.1103/PhysRevLett.109.012001

59. F.D. Aaron et al., H1 Collaboration. JHEP. 1209, 061 (2012). arXiv:1206.7007. doi:10.1007/JHEP09(2012)061

60. I.I. Bigi, M.A. Shifman, N. Uraltsev, A. Vainshtein, Phys. Rev. D 50, 2234 (1994). arXiv:hep-ph/9402360. doi:10.1103/PhysRevD. 50.2234

61. M. Beneke, V.M. Braun, Nucl. Phys. B. 426, 301 (1994). arXiv:hep-ph/9402364. doi:10.1016/0550-3213(94)90314-X

62. M. Beneke, Phys. Rept. 317, 1 (1999). arXiv:hep-ph/9807443. doi:10.1016/S0370-1573(98)00130-6

63. O. Zenaiev, Charm production and QCD analysis at HERA and LHC. Ph.D. Thesis, Hamburg University, 2015, Report DESYTHESIS-2015-012

64. A.D. Martin, W.J. Stirling, R.S. Thorne, G. Watt, Eur. Phys. J. C 70, 51 (2010). arXiv:1007.2624. doi:10.1140/epjc/ s10052-010-1462-8 